Measurement Science and Technology

\title{
Femtosecond X-rays from Thomson scattering using laser wakefield accelerators
}

\author{
P. Catravas, E. Esarey and W.P. Leemans \\ Center for Beam Physics, Accelerator and Fusion Research Division \\ Ernest Orlando Lawrence Berkeley National Laboratory \\ One Cyclotron Road, University of California, Berkeley CA 94720
}

\begin{abstract}
The possibility of producing femtosecond x-rays through Thomson scattering high power laser beams off laser wakefield generated relativistic electron beams is discussed. The electron beams are produced with either a self-modulated laser wakefield accelerator (SM-LWFA) or through a standard laser wakefield accelerator (LWFA) with optical injection. For a SMLWFA (LWFA) produced electron beam, a broad (narrow) energy distribution is assumed, resulting in X-ray spectra that are broadband (monochromatic). Designs are presented for 3-100 fs X-ray pulses and the expected flux and brightness of these sources are compared.
\end{abstract}

Keywords: X-ray, femtosecond, laser, electron beam, Thomson scattering, diffraction, laser wakefield acceleration. 


\section{INTRODUCTION}

There are many different approaches for the generation of femtosecond x-ray pulses [1], one of them being Thomson scattering (TS) [2] an intense laser off an intense electron beam [3]- [15]. The first demonstration of the generation of sub-picosecond duration x-ray pulses using $90^{\circ} \mathrm{TS}$ was implemented at the Beam Test Facility of the Advanced Light Source at Lawrence Berkeley National Laboratory (LBNL) [7]- [9]. The generated X-ray pulse duration of 300 fs (FWHM) was determined by the convolution between the laser pulse duration (100 fs) and the crossing time of the laser across the tightly focused electron beam (200 - $250 \mathrm{fs})$. The number of x-rays and peak brightness of the $90^{\circ} \mathrm{TS}$ source experiments at LBNL was in part limited by the fact that the laser beam only interacted with about a 100 fs long electron beam slice (or $0.3 \%$ of all the available electrons), as well as the relatively high transverse emittance of the electron beam and low peak laser power used in the experiment. To increase the photon yield and source brightness, high quality femtosecond electron bunches are needed. Such electron bunches could then be used to produce femtosecond x-rays through $180^{\circ}$ laser backscattering or through Bremsstrahlung in a thin, solid target [15].

In this paper, we discuss the use of electron beams produced with laser wakefield accelerators (LWFAs) [16] for TS sources. The characteristic scale length of the accelerating field in a plasma-based accelerator [16] is the plasma wavelength, $\lambda_{p}[\mathrm{~m}] \simeq 3.3 \times 10^{4} n_{p}^{-1 / 2}\left[\mathrm{~cm}^{-3}\right]$, where $n_{p}$ is the plasma density. In the self-modulated laser wakefield accelerator (SMLWFA) [17]- [18], energies up to $100 \mathrm{MeV}$ have been demonstrated in several experiments [19]- [27], however, with large (100\%) energy spread, since the electrons are self-trapped from the background plasma. In the standard LWFA (typically $\lambda_{p} \sim 100 \mu \mathrm{m}$ ) electrons are not self-trapped and the production of electron beams with low momentum spread and good pulse-to-pulse energy stability requires femtosecond electron bunches to be injected with femtosecond synchronization with respect to the plasma wake. Although conventional electron sources (photocathode or thermionic RF guns) have achieved sub-picosecond electron 
bunches, the requirements for injection into plasma-based accelerators are presently beyond the performance of these conventional electron sources. Novel schemes which rely on laser triggered injection of plasma electrons into their own plasma wake have been proposed to generate the required femtosecond electron bunches [28]- [32].

We present designs of two novel sources of ultrashort x-ray pulses using TS from relativistic electron beams produced with LWFAs. In Sec. 2, analytic expressions for TS including broadening from an arbitrary energy distribution are presented. These expressions are valid when the number of periods in the interaction region is large and provide a new and efficient way to evaluate the broadened spectral flux density for the wide range of possible energy distributions which LWFAs can produce. The limiting case of a narrow Gaussian distribu-

tion is shown to agree with standard formulae, and the analysis is applied in the case of 100\% energy spread, where standard formulae do not apply. In Sec. 3, TS designs using electron bunches produced via the SMLWFA and a standard LWFA with optical injection are compared. The SMLWA yields large amounts of charge having large energy spread due to uncontrolled trapping, and can provide X-ray flux over a large BW in a $<100$ fs pulse. The colliding pulse all-optical injector [28]- [30], which utilizes optical methods for triggering the trapping of electrons, holds the promise to produce low emittance electron bunches with low energy spread, which can be used to generate high brightness X-ray pulses with a few fs pulse length.

\section{TS SPECTRUM FOR ARBITRARY ENERGY DISTRIBUTION}

In general, the computation of spectral flux density from an electron beam, including realistic spreads in beam parameters, is performed through convolution of the electron distribution with the single electron spectral flux density. For a normalized electron distribution function, $f\left(\gamma, \theta_{e}\right)$, the resulting spectral flux density of the TS radiation from an electron beam is given by

$$
\frac{d^{2} I_{T}}{d \omega d \Omega}(\theta, \omega)=\int d \theta_{e} d \gamma f\left(\theta_{e}, \gamma\right) \frac{d^{2} I}{d \omega d \Omega}\left(\theta-\theta_{e}, \gamma, \omega\right),
$$


where $d^{2} I(\theta, \gamma, \omega) / d \omega d \Omega$ is the energy radiated by a single electron per unit frequency and solid angle, $\theta$ is the observation angle with respect to the longitudinal axis, $\omega$ the frequency of the TS radiation, $\theta_{e}$ is the angle the electron trajectory with respect to the longitudinal axis $\left(\theta_{e}^{2} \ll 1\right.$ is assumed), and $\gamma$ is the electron energy.

The electron beams characteristic of LWFA designs under current development provide strongly contrasting cases of inhomogeneous broadening. When the electron energy distribution is narrow, standard formulae [3]- [9], [15] can be used to evaluate the energy spread contribution to figures of merit such as on-axis flux and brightness. Such is the case for the colliding pulse optical injection in a standard LWFA. However, when the energy distribution is broad, such as the $100 \%$ energy spread of the SMLWFA, the standard formulae cannot be applied. They become inapplicable because the on-axis frequency, $4 \gamma^{2} \omega_{0}$, $\left(a_{0} \ll 1, \theta=0\right)$ can change by up to three orders of magnitude across the energy distribution, accompanied by a factor of thirty change in the on-axis opening angle, $1 /\left(\gamma \sqrt{N_{0}}\right)$. Here, $a_{0} \simeq 8.5 \times 10^{-10} \lambda_{0}[\mu \mathrm{m}] I^{1 / 2}\left[\mathrm{~W} / \mathrm{cm}^{2}\right]$ is the normalized vector potential of the laser pulse (laser strength parameter), $I$ is the laser pulse intensity, $\lambda_{0}$ is the laser wavelength, $\omega_{0}=2 \pi c / \lambda_{0}$ is the laser frequency, and $N_{0}$ is the number of periods of the laser pulse with which the electron interacts. When $a_{0}^{2}$ is not small compared with unity, the situation is even more complex, as there is substantial overlap between harmonics radiated by lower energy electrons in the distribution and radiation at the fundamental by higher energy electrons.

This problem can be overcome by noticing that a very large number of periods is available in the interaction region (up to 1000's) in TS designs, so that the intrinsic spectral line broadening at any fixed angle is essentially a delta function compared with the contribution from the energy distribution for realistic parameters. In this case, analytic expressions can be obtained for spectral flux density which are valid for arbitrary energy distributions, including the $100 \%$ energy distribution of the SMLWA. 


\section{A. Single electron spectrum}

The general form of the energy radiated by a single electron per unit frequency, $d \omega$, per unit solid angle, $d \Omega$, derived by integrating the Lienard Wiechert potentials, can be found in reference [4]. Here we consider the small $a_{0}$ limit $\left(a_{0}^{2} \ll 1\right)$, where the main contribution to the radiation comes from the fundamental. For typical experimental parameters and regimes of interest, the electron beam energy satisfies $\gamma^{2} \gg 1$ and the angle of observation is small $\left(\theta^{2} \ll 1\right)$. Then, the TS spectral flux density simplifies to

$$
\frac{d^{2} I}{d \omega d \Omega} \simeq r_{e} m c\left(\frac{\omega}{4 \gamma^{2} \omega_{0}}\right)^{2} \gamma^{2} N_{0}^{2} a_{0}^{2} R\left(\omega, \omega_{0}\right),
$$

where $r_{e}=e^{2} / m c^{2}$ is the classical electron radius, $m$ is the electron mass, $c$ is the speed of light, and the spectral flux density has been evaluated in the plane perpendicular to that of the laser polarization.

The resonance function, $R\left(\omega, \omega_{0}\right)$ determines many of the defining characteristics of Thomson scattered radiation:

$$
R\left(\omega, \omega_{0}\right)=\left(\frac{\sin \bar{k} L / 2}{\bar{k} L / 2}\right)^{2}
$$

where

$$
\bar{k}=k\left(1+\gamma^{2} \theta^{2}\right) /\left(4 \gamma^{2}\right)-k_{0}
$$

$L=N_{0} \lambda_{0}$ is the length of the interaction region, $k=\omega / c$ is the radiation wavenumber, and

$k_{0}=\omega_{0} / c$ is the laser wavenumber. The condition $\bar{k}=0$ corresponds to a Doppler shifted resonant frequency of

$$
\omega_{r} \simeq 4 \gamma^{2} \omega_{0} /\left(1+\gamma^{2} \theta^{2}\right)
$$

or, equivalently, a resonant energy of

$$
\gamma_{r}^{2} \simeq \frac{\omega / 4 \omega_{0}}{\left(1-\omega \theta^{2} / 4 \omega_{0}\right)}
$$


Of particular interest to TS using laser wakefield electron injectors is the behavior for a large number of periods, $N_{0}$. In the limit $N_{0} \rightarrow \infty$,

$$
R \rightarrow \Delta \omega_{r} \delta\left(\omega-\omega_{r}\right)=\Delta \gamma_{r} \delta\left(\gamma-\gamma_{r}\right)
$$

where $\Delta \omega_{r}=\omega_{r} / N_{0}$ is the spectral width, which corresponds to $\Delta \gamma_{r}=2 \gamma_{r}^{3} \omega_{0} / N_{0} \omega_{r}$.

\section{B. Spectrum for the SMLWA bunch}

To include the effect of energy spread, the spectral flux density is integrated over electron energy spectrum, $f(\gamma)$,

$$
\frac{d^{2} I_{T}}{d \omega d \Omega} \simeq \int d \gamma f(\gamma) \frac{d^{2} I}{d \omega d \Omega}
$$

Beam emittance is neglected since the angular width of the spectrum is much broader than typical beam divergence for photon energies of interest (see Fig. 2c). Assume $f(\gamma)$ is slowly varying compared to $R\left(\omega, \omega_{r}\right)$ for fixed $\omega$. Integrating over the delta function approximation for $R\left(\omega, \omega_{r}\right)$ in frequency (energy) yields the following analytic form for the energy integrated spectrum

$$
\frac{d^{2} I_{T}}{d \omega d \Omega} \simeq \frac{r_{e} m c}{16} N_{0} a_{0}^{2}\left(\frac{\omega}{\omega_{0}}\right)^{3 / 2} f\left(\gamma=\left(\omega / 4 \omega_{0}\right)^{1 / 2}\right)
$$

Of particular interest is the photon flux and brightness of the TS radiation. Assuming that the collection angle $\theta_{d}$ is small, $\theta_{d}<(\Delta \omega / \omega)^{1 / 2} / \gamma<(1 / N)^{1 / 2} / \gamma$, so that the intensity distribution is flat over the solid angle $\Delta \Omega_{d}=\pi \theta_{d}^{2}$, the number of photons intercepted in a small bandwidth $\Delta \omega$ and solid angle $\Delta \Omega_{d}$ is

$$
N_{T}=\frac{1}{\hbar} \frac{d^{2} I_{T}}{d \omega d \Omega} \frac{\Delta \omega}{\omega} \pi \theta_{d}^{2} \simeq \frac{\alpha_{f}}{16} N_{0} a_{0}^{2}\left(\frac{\omega}{\omega_{0}}\right)^{3 / 2} f\left(\gamma=\left(\omega / 4 \omega_{0}\right)^{1 / 2}\right) \frac{\Delta \omega}{\omega} \pi \theta_{d}^{2}
$$

The average flux in the collection angle $\theta_{d}$ and with bandwidth $\Delta \omega, F_{a v}$, in photons per second, is $N_{T}$ multiplied by the repetition rate, $f_{r e p}$, of the laser pulse/electron beam interaction, i.e., $F_{a v}=N_{T} f_{r e p}$. The average source brightness (in photons $/ \mathrm{s} / \mathrm{mm}^{2} / \mathrm{mrad}^{2} / 0.1 \%$ BW) is given by 


$$
B_{a v}=\frac{F_{a v}}{(2 \pi)^{2} r_{b}^{2} \theta_{d}^{2}} \simeq \frac{\alpha_{f} N_{0} a_{0}^{2}}{64 \pi r_{b}^{2}}\left(\frac{\omega}{\omega_{0}}\right)^{3 / 2} f\left(\gamma=\left(\omega / 4 \omega_{0}\right)^{1 / 2}\right) \frac{\Delta \omega}{\omega}
$$

where $r_{b}$ is the electron bunch radius. The peak flux and brightness are, respectively, $F_{p k}=$ $F_{a v} /\left(\tau_{x} f_{r e p}\right)$ and $B_{p k}=B_{a v} /\left(\tau_{x} f_{r e p}\right)$, where $\tau_{x}$ is the x-ray pulse duration, which is assumed to be approximately equal to the electron bunch duration.

\section{TS spectrum for narrow energy distribution}

For an electron bunch with a narrow energy spread $\Delta \gamma / \gamma_{0} \ll 1$ about a mean energy, $\gamma_{0}$, and for a narrow distribution in beam angle with spread $\Delta \theta=\epsilon_{n} / \gamma r_{b} \ll 1$, where $\epsilon_{n}$ is the normalized emittance and $r_{b}$ the beam radius, estimates for flux and brightness have been derived [4], [27]. In particular, the total number of photon scattered per laser-electron bunch interaction, into a small bandwidth $\Delta \omega / \omega \ll 1$, is approximately

$$
N_{T}=2 \pi \alpha N_{0} a_{0}^{2} \frac{\Delta \omega}{\omega} N_{b} F_{\text {coll }}
$$

assuming $a_{0}^{2} \ll 1$, where $N_{b}$ is the number of electrons per bunch interacting with the laser

pulse. Here, $F_{\text {coll }}$ is a factor determined by the collection angle $\theta_{d}$ of the X-ray optics, i.e., $F_{\text {coll }}=\theta_{d}^{2} /\left(\theta_{d}^{2}+\theta_{T}^{2}\right)$ with

$$
\theta_{T}^{2} \simeq\left[(\Delta \omega / \omega)^{2}+(\Delta \omega / \omega)_{0}^{2}+(\Delta \omega / \omega)_{\epsilon}^{2}+(\Delta \omega / \omega)_{i}{ }^{2}\right]^{1 / 2} / \gamma^{2}
$$

where $(\Delta \omega / \omega)_{0}=1 / N_{0},(\Delta \omega / \omega)_{\epsilon}=\epsilon_{n}{ }^{2} / r_{b}{ }^{2}$ and $(\Delta \omega / \omega)_{i}=2 \Delta \gamma / \gamma$ represent the contributions to the bandwidth from the finite interaction length, the beam emittance and energy spread, respectively. The average flux is $F_{a v}=N_{T} f_{r e p}$ and the average brightness is

$$
B_{a v}=\frac{N_{T} f_{r e p}}{(2 \pi)^{2} \sigma_{r}^{2} \sigma_{\theta}^{2}}
$$

where $\sigma_{r} \simeq r_{b}$ and $\sigma_{\theta} \simeq \theta_{T}$ are the rms source size and opening angle of the radiation, respectively, and $r_{b} \simeq r_{0}$ has been assumed, where $r_{0}$ is the laser pulse radius. 


\section{EXAMPLES BASED ON LWFAS}

\section{A. Self-Modulated Laser Wakefield Accelerator}

The SMLWA provides short $(<100 \mathrm{fs})$ high charge $\left(>10^{10}\right.$ electrons) bunches having a broad energy distribution (100\%). Thus TS using a SMLWA injector will be characterized by high photon flux with and broad bandwidth. In the SMLWA, an instability initiated at the front of a laser pulse leads to increasingly strong self modulation of the laser pulse envelope at $\lambda_{p}$ periodicity and resonant enhancement of the wakefield. The requirements for this instability are a laser pulse long compared with $\lambda_{p}$ and powers exceeding the threshold for relativistic self-focussing. The strong enhancement of wakefield strength over the standard LWFA leads to self-trapping of plasma electrons, while the relativistic focussing increases the acceleration distance, allowing high electron energies to be reached with a high charge per bunch. Bunch lengthening due to space charge effects [33] can be avoided by minimizing the propagation distance of the electron bunch before the TS interaction, thus keeping the

electron bunch length as close as possible to the laser pulse length. Shorter drive pulse lengths combined with higher plasma densities can lead to higher X-ray brightness and shorter electron beam bunch lengths. That is, decreasing the laser pulse length $L=c \tau_{L}$ requires increasing plasma density to satisfy $L>\lambda_{p}$, and the wake axial electric field scales roughly as $E_{z} \sim \sqrt{n_{0}}$. Uncontrolled trapping leads to a broad energy distribution of the form [21]- [27]

$$
f(\gamma)=f_{0} \exp \left(-\gamma / \gamma_{0}\right),
$$

where $f_{0}$ and $\gamma_{0}$ are constants.

Empirical parameters for the LBNL SMLWA are as follows [27]: The electron distribution for a SMLWA having a $10 \mathrm{~Hz}$ driving pulse of $50 \mathrm{fs}$ and $a_{0} \sim 1$ and plasma density of a few $10^{19} \mathrm{~cm}^{-3}$, as measured with a bending magnet, is

$$
f_{g}(\gamma)=0.24 e^{-0.3 \gamma}
$$



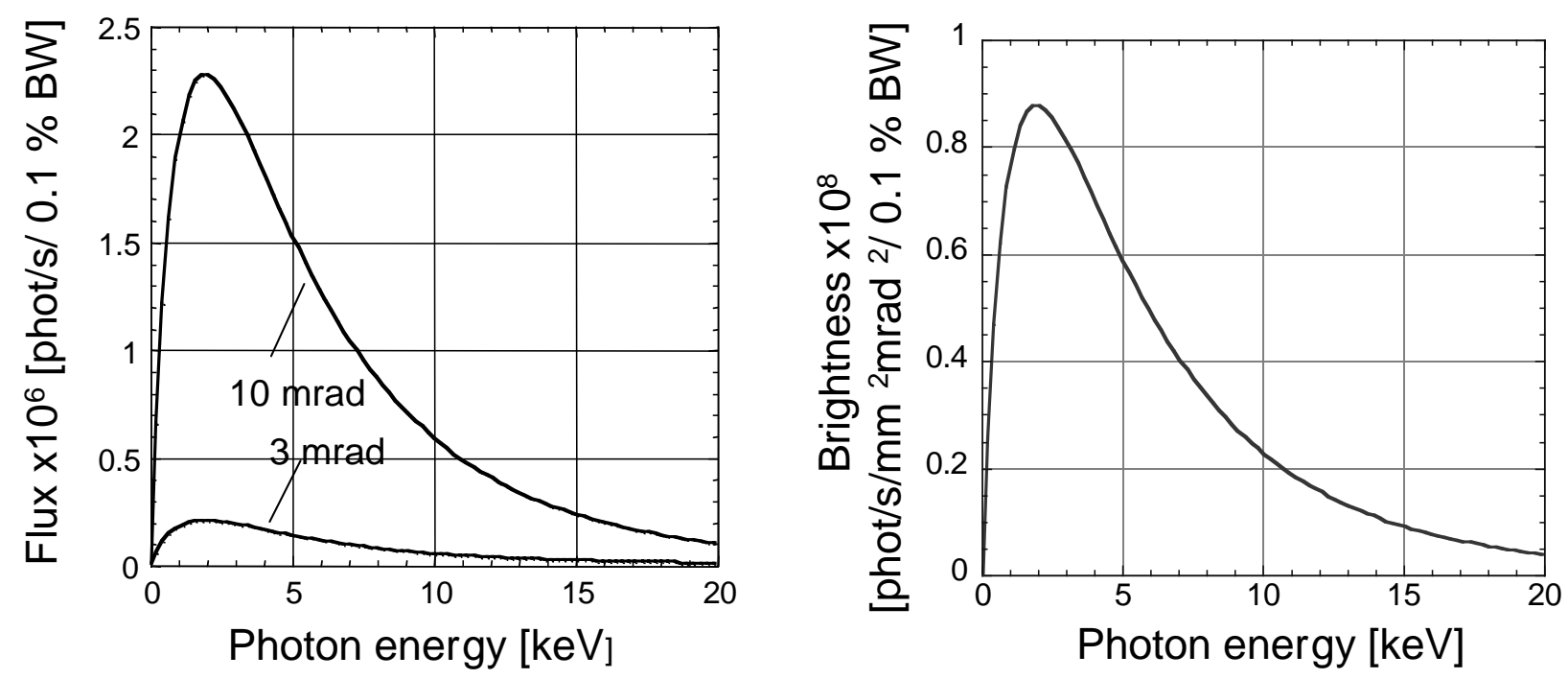

FIG. 1. The average flux into 3 and $10 \mathrm{mrad}$ collection angles and average brightness for the SMLWA-based TS design (see Table 2, col. 2 for parameters) shows the influence of the high charge per bunch $(\sim 5 \mathrm{nC})$ and broad energy distribution.

The total charge is at least $5 \mathrm{nC}$ per bunch, or $3 \times 10^{10}$ particles.

Using these electron beam parameters, the following TS source may be designed. A 600 mJ, 1.4 ps laser pulse with a wavelength of $800 \mathrm{~nm}$ and a rep rate of $10 \mathrm{~Hz}$ is focussed to a spot size of 6 microns. With a corresponding $a_{0}$ of 0.7 , about $3 \times 10^{11}$ photons per second are radiated in all frequencies and all angles. Brightness as a function of photon energy in photons $/ \mathrm{s} / \mathrm{mm}^{2} \mathrm{mrad}^{2} / 0.1 \% \mathrm{BW}$ at a $10 \mathrm{~Hz}$ rep rate, calculated with Eq. (11), is shown in Fig. 1 1. The average brightness for these parameters peaks between 2 and $3 \mathrm{keV}$ and has a total bandwidth of about $10 \mathrm{keV}$. As the result of the use of an ultrashort laser pulse, the peak brightness $\left(B_{p k}=B_{a v} /\left(\tau_{x} f_{r e p}\right) \sim 3-4 \times 10^{19}\right.$ photons $/ \mathrm{s} / \mathrm{mm}^{2} \mathrm{mrad}^{2} / 0.1 \%$ BW) is 12 orders of magnitude higher than the average brightness for these parameters. The parameters and X-ray characteristics are summarized in Table 1, column 2.

The full spectral flux density over a range of $20 \mathrm{keV}$ in photon energy and $50 \mathrm{mrad}$ observation angle is shown in Fig. 2c, accompanied by the electron energy distribution (Fig. 2a), computed from a generalization of Eq. (11) to account for off-axis angles. The higher the on-axis photon energy corresponding to each slice in energy the lower the amplitude. Beam emittance is about $1 \pi \mathrm{mm}$ mrad. Note that for the corresponding divergences of $\sim 10$ 
a)

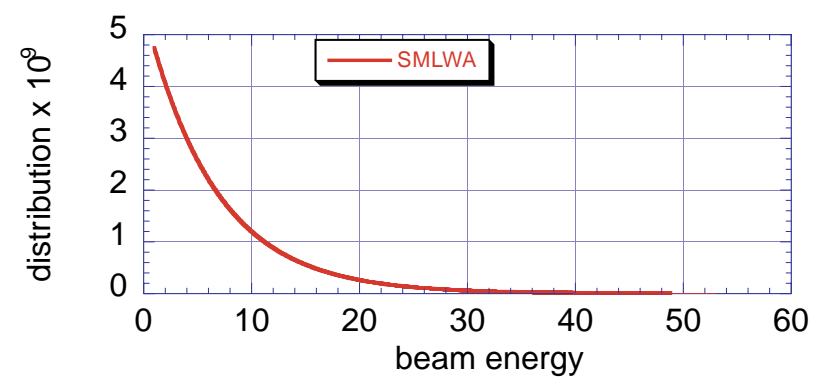

b)

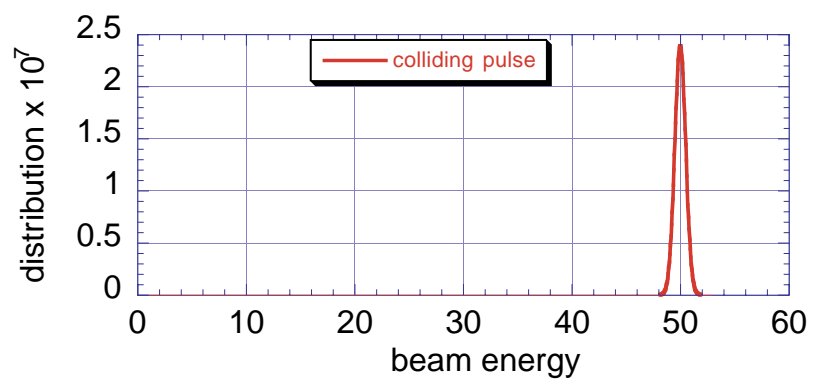

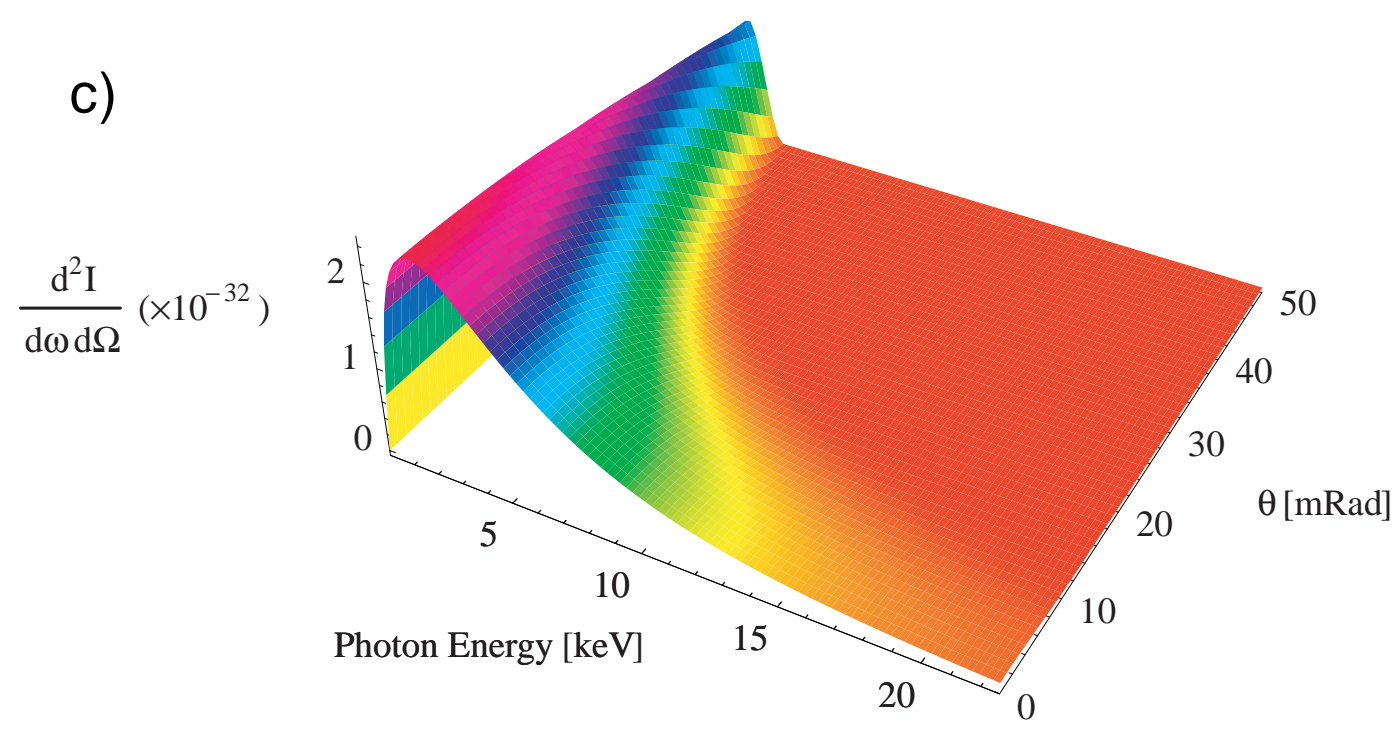

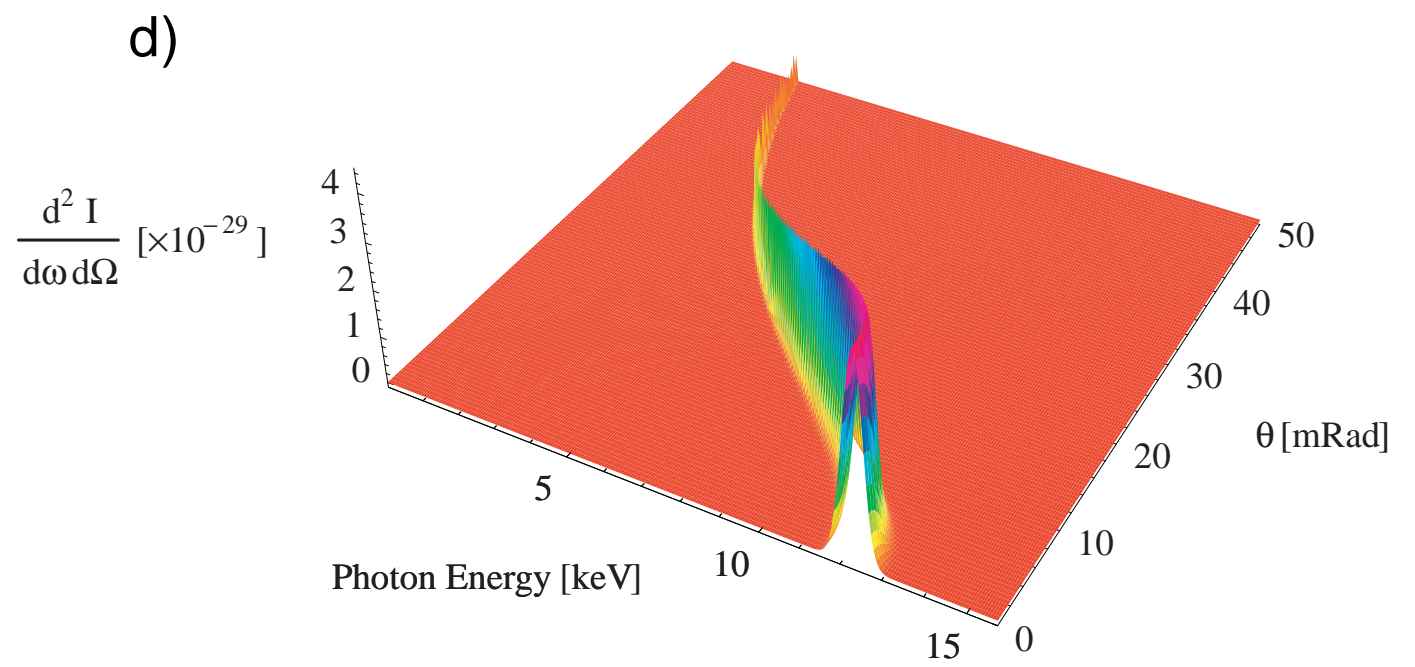

FIG. 2. Energy distribution (a) and spectral flux density (c) for SMLWA; energy distribution (b) and spectral flux density (d) for colliding pulse for the parameters in Table 1. The integral of the distributions are $3 \times 10^{10}$ and $3 \times 10^{7}$ electrons per bunch for SMLWA and colliding pulse, respectively. The intensity distributions illustrate the contrast between the two injection schemes in scattered X-ray bandwidth and spatial collimation at a fixed observation frequency. 
mrad, divergence broadening can be neglected for the bulk of the emissions (photon energies less than $15 \mathrm{keV})$.

\section{B. Colliding Pulse All-Optical Injector}

In this section, we discuss generating near-monochromatic x-rays using TS off electron bunches produced with the colliding pulse laser-plasma electron source for generating truly femtosecond electron bunches [28]- [30]. The source generates ultrashort electron bunches by using laser pulses to dephase background plasma electrons undergoing fluid oscillations in a standard LWFA plasma wake. This colliding pulse scheme has the ability to produce femtosecond electron bunches with low fractional energy spreads using relatively low injection laser pulse intensities compared to the pump laser pulse. $\left(a_{i n j}^{2} \ll a_{p u m p}^{2} \sim 1\right.$, where $a=e A / m c^{2}$ is the normalized vector potential of the pulse).

The colliding pulse optical injection scheme employs three short laser pulses: an intense $\left(a_{0}^{2} \simeq 1\right)$ pulse (denoted by subscript 0$)$ for plasma wake generation, a forward going injection pulse (subscript 1), and a backward going injection pulse (subscript 2). The frequency, wavenumber, and normalized intensity are denoted by $\omega_{i}, k_{i}$, and $a_{i}(i=0,1,2)$. Furthermore, $\omega_{1}=\omega_{0}, \omega_{2}=\omega_{0}-\Delta \omega(\Delta \omega \geq 0)$, and $\omega_{0} \gg \Delta \omega \gg \omega_{p}$ are assumed such that $k_{1}=k_{0}$, and $k_{2} \simeq-k_{0}$. The pump pulse generates a plasma wake with phase velocity near the speed of light $\left(v_{p 0} \simeq c\right)$. When the injection pulses collide (some distance behind the pump) they generate a slow ponderomotive beat wave with a phase velocity $v_{p b} \simeq \Delta \omega / 2 k_{0} \ll c$. During the time in which the two injection pulses overlap, a two-stage acceleration process can occur, i.e., the slow beat wave injects plasma electrons into the fast wakefield for acceleration to high energies. Injection and acceleration can occur at low densities $\left(\lambda_{p} / \lambda \sim 100\right)$, thus

allowing for high single-stage energy gains, with normalized injection pulse intensities of $a_{1} \sim a_{2} \sim 0.2$. Furthermore, the colliding pulse concept offers detailed control of the injection process: the injection phase can be controlled via the position of the forward injection pulse, the beat phase velocity via $\Delta \omega$, the injection energy via the pulse amplitudes, and 
the injection time (number of trapped electrons) via the backward pulse duration.

As an example, for a plasma density of $N_{b}=7 \times 10^{17} \mathrm{~cm}^{-3}$, the maximum trapping fraction corresponds to a bunch number of $N_{b} \sim 0.5 \times 10^{7}$ electrons. Note that the bunch number can be increased by increasing the laser spot sizes (i.e., laser powers). For example, when the laser spot sizes are doubled $r_{s i}=30 \mu \mathrm{m}$ the number of trapped electrons increases to $\sim 1.5 \times 10^{7}$ and the normalized transverse emittance increases to $\sim 3.9 \mathrm{~mm}-\mathrm{mrad}$. Estimates indicate that space charge effects can be neglected while the bunch remains inside the plasma [29] and can be minimized for sufficiently high energy electron beams [33].

In the TS example summarized in column 1 of Table 1, a 0.8 micron laser with 500 mJ energy and 1 ps duration focussed to a 6 micron spot is scattered off of a $25 \mathrm{MeV}$ electron beam with a few fs bunch length, assuming the electron beam parameters from simulations discussed in the previous section, designed for an on-axis photon energy of 12.4 $\mathrm{keV}$. With a rep rate of $10 \mathrm{~Hz}$, and a $1 \mathrm{mrad}$ collection angle, the average brightness is about $10^{8}$ photons $/ \mathrm{s} / \mathrm{mm}^{2} \mathrm{mrad}^{2} / 0.1 \%$ BW. Due to the low contributions of inhomogeneous broadening, the peak brightness of $\left(5 \times 10^{21}\right.$ photons $\left./ \mathrm{s} / \mathrm{mm}^{2} \mathrm{mrad}^{2} / 0.1 \% \mathrm{BW}\right)$ is two orders of magnitude higher than for the SMLWA in spite of 3 orders of magnitude lower total flux into all angles and frequencies. From this example we conclude that this unique source, capable of producing truly femtosecond pulses, should have sufficient flux and brightness to perform pump probe type experiments.

The full spatial and spectral characteristics are shown in Fig. 2d, along with the energy distribution (Fig. 2b). Note that this profile can be thought of as a composite slice of the SMLWA case. The energy distribution for the SMLWA is similar to a weighted sum of narrow energy slices, and the topography of the spectral flux density of the SMLWA arises from a sum of weighted contributions similar to Fig. $2 \mathrm{~d} d$, each having a different on-axis frequency. Because $N_{0}$ is large, the spectral flux density contains a strong signature of the energy distribution in both designs and can be used as a diagnostic. 


\begin{tabular}{|c|c|c|c|}
\hline Parameter & Colliding Pulse & SMLWA & BTF \\
\hline Laser wavelength $\lambda_{L}$ & $0.8 \mu \mathrm{m}$ & $0.8 \mu \mathrm{m}$ & $0.8 \mu \mathrm{m}$ \\
\hline Laser pulse energy $U_{L}$ & $0.5 \mathrm{~J}$ & $0.6 \mathrm{~J}$ & $0.04 \mathrm{~J}$ \\
\hline Laser pulse duration $(\mathrm{FWHM}) \tau_{L}$ & $1 \mathrm{ps}$ & $1.4 \mathrm{ps}$ & $100 \mathrm{fs}$ \\
\hline Electron beam energy $\gamma$ & 50 & Exp. Distribn. & 98 \\
\hline Number of electrons $N_{b}$ & $3 \times 10^{7}$ & $3 \times 10^{10}$ & $8 \times 10^{9}$ \\
\hline Electron bunchlength (FWHM) $\tau_{b}$ & $3 \mathrm{fs}$ & $100 \mathrm{fs}$ & $30 \mathrm{ps}$ \\
\hline Electron spot size (FWHM) $r_{b}$ & $6 \mu \mathrm{m}$ & $6 \mu \mathrm{m}$ & $90 \mu \mathrm{m}$ \\
\hline Normalized emittance $\epsilon_{N}$ & $1 \mathrm{~mm}-\mathrm{mrad}$ & $1 \mathrm{~mm}-\mathrm{mrad}$ & $30 \mathrm{~mm}-\mathrm{mrad}$ \\
\hline Bandwidth $\delta \omega / \omega$ & $10^{-3}$ & $10^{-3}$ & $10^{-3}$ \\
\hline Collection angle & $1 \mathrm{mrad}$ & $3 \mathrm{mrad}$ & $1 \mathrm{mrad}$ \\
\hline Repetition rate & $10 \mathrm{~Hz}$ & $10 \mathrm{~Hz}$ & $2 \mathrm{~Hz}$ \\
\hline Flux $(\mathrm{ph} / \mathrm{s} / 0.1 \% \mathrm{BW})$ in coll. angle & $5 \times 10^{4}$ & $2 \times 10^{5}$ & $6 \times 10^{2}$ \\
\hline Ave. brightness (ps/s/mm² $\left.-\mathrm{mrad}^{2} / 0.1 \% \mathrm{BW}\right)$ & $1.5 \times 10^{8}$ & $9 \times 10^{7}$ & $3 \times 10^{3}$ \\
\hline Peak brightness $\left(\mathrm{ps} / \mathrm{s} / \mathrm{mm}^{2}-\mathrm{mrad}^{2} / 0.1 \% \mathrm{BW}\right)$ & $5 \times 10^{21}$ & $10^{20}$ & $10^{16}$ \\
\hline Tot. no. photons/s (all freq., all angles) & $2 \times 10^{8}$ & $3 \times 10^{11}$ & $2 \times 10^{7}$ \\
\hline X-ray pulselength & $3 \mathrm{fs}$ & $<100$ fs & $300 \mathrm{fs}$ \\
\hline X-ray photon energy $(\mathrm{keV})$ & $12.4 \mathrm{keV}$ & $\begin{array}{l}\text { Broadband, } \\
\max \text { at } 2-3 \mathrm{keV}\end{array}$ & $30 \mathrm{keV}$ \\
\hline
\end{tabular}

TABLE I. Comparison of TS designs using laser wakefield accelerators and experimental results with an RF accelerator.

\section{CONCLUSION}

Laser wakefield accelerators provide electron bunches suitable for the production of ultrashort, high brightness x-ray pulses through $180^{\circ}$ Thomson scattering. To date the study of 
ultrafast processes has largely relied on femtosecond optical pulses from mode-locked lasers. Since x-rays interact with core electronic levels and hence are effective structural probes, the availability of femtosecond $\mathrm{x}$-ray pulses and the intrinsic synchronization between laser and x-ray pulses would make it possible to directly probe changes in atomic structure on ultrafast time scales.

The self-modulated laser wakefield accelerator provides high charge $(5 \mathrm{nC})$ in bunches of less than 100 fs at 100\% energy spread (the bulk of the distribution is at energies of a couple of $\mathrm{MeV}$ with a exponential tail extending out to tens of $\mathrm{MeV}$ ). The TS spectrum and spatial profile is characteristically broad. Based on current measured values for the such electron bunches, a design having $a_{0}<1$ which sets the bulk of the photon energies in the range of a few $\mathrm{keV}$ has been described. Peak brightnesses are predicted to exceed $10^{19}$ photons $/ \mathrm{s} / \mathrm{mm}^{2} \mathrm{mrad}^{2} / 0.1 \% \mathrm{BW}$.

The proposed colliding pulse optical injection scheme has the ability to produce electron bunches of a few fs, having low energy spread and emittance. While charge levels are lower than for the SMLWA, low energy spread and divergence enables an increase of two orders of magnitude in peak brightness.

Although summarizing source performance using a single number (e.g. peak brightness) can be useful for some applications, proper evaluation must be made based on the type of experiment and its requirements, as well as on the ease of implementation and available infrastructure. These laser-plasma electron beam sources offer some unique advantages: (1) TS based sources can produce tunable ultra-short x-ray pulses using relatively low energy electron beams. (2) The source parameters such as photon energy, brightness, and bandwidth, are controllable through electron beam and laser parameters. For example, rapid polarization and wavelength control is possible through the laser polarization and wavelength tuning, respectively. (3) The pulse length is controllable through the laser pulse length, electron bunch length, and interaction geometry. (4) The methods provide perfect synchronization between the laser pulse, electron bunch, and x-ray pulse.

These laser-plasma sources of x-rays are of table-top size. The major equipment required 
for such a source is a high power, chirp-pulse amplification laser system, which is becoming commonplace [34]. However, for all laser-plasma sources, laser repetition rate and average power have been some of the main limitations. Future research into the development of high average power lasers, as well as optical storage cavities for ultra-short pulses, would have a tremendous impact on the scientific reach of these laser-plasma based sources.

\section{ACKNOWLEDGMENTS}

This work was supported by the Department of Energy under Contract No. DE-AC-0376SF0098. 


\section{REFERENCES}

[1] See, for example, Special Issue on New Ultrafast X-ray Sources and Their Applications, Edited by J.C. Gauthier, Les Comptes Rendus de l'Académie des Sciences, Série IV, 1 $(2000)$.

[2] F.R. Arutyumain and V.A. Tumanian, Phys. Lett. 4, 173 (1963); R.H. Milburn, Phys. Rev. Lett., 10, 75 (1963); E.S. Sarachik and G.T. Schappert, Phys. Rev. D, 1, 2738 (1970).

[3] P. Sprangle, A. Ting, E. Esarey and A. Fisher, J. Appl. Phys. 72 (1992) 5032; P. Sprangle and E. Esarey, Phys. Fluids B 4 (1992) 2241.

[4] E. Esarey, S.K. Ride and P. Sprangle, Phys. Rev. E 48 (1993) 3003.

[5] S.K. Ride, E Esarey and M. Baine. Phys. Rev. E 52 (1995) 5425; E. Esarey, S.K. Ride, M. Baine, A. Ting and P. Sprangle, Nucl. Instr. and Meth. A 375 (1996) 545.

[6] K.J. Kim, S. Chattopadhyay and C.V. Shank, Nucl. Instr. and Meth. A 341 (1994) 351.

[7] R.W. Schoenlein, W.P. Leemans, A.H. Chin, P. Volfbeyn, T.E. Glover, P. Balling, M. Zolotorev, K.J. Kim, S. Chattopadhyay and C.V. Shank, Science 274 (1996) 236.

[8] W.P. Leemans, R.W. Schoenlein, P. Volfbeyn, A.H. Chin, T.E. Glover, P. Balling, M. Zolotorev, K.J. Kim, S. Chattopadhyay and C.V. Shank, Phys. Rev. Lett. 77 (1996) 4182.

[9] W.P. Leemans, R.W. Schoenlein, P. Volfbeyn, A.H. Chin, T.E. Glover, P. Balling, M. Zolotorev, K.J. Kim, S. Chattopadhyay and C.V. Shank, IEEE J. Quantum Electron. 33 (1997) 1925.

[10] Ting, A., R. Fischer, A. Fisher, K. Evans, R. Burris, J. Krall, E. Esarey and P. Sprangle, J. Appl. Phys. 78, 575 (1995); A. Ting, private communication.

[11] F. Glotin, J.-M. Ortega, R. Prazeres, G. Devanz and O. Marcouille, Phys. Rev. Lett., 
77, 3130 (1996).

[12] F.E. Carroll, J. W. Waters, R. H. Traeger, M. H. Mendenhall, W.-W. Clark, C. A. Brau, SPIE Proceedings Vol. 3614: 139-146, January 1999.

[13] G. Krafft, Jefferson Laboratory, private communication (1999).

[14] I.V. Pogorelsky, I. Ben-Zvi, T. Hirose, S. Kashiwagi, V. Yakimenko, K. Kusche, P. Siddons, J. Skaritka, T. Kumita, A. Tsunemi, T. Omori, J. Urakawa, M. Washio, K. Yokoya, T. Okugi, Y. Liu, P. He, D. Cline, Phys. Rev. Spec. Topics Accel. Beams, 3 $090702(2000)$.

[15] W. Leemans, S. Chattopadhyay, E. Esarey, A. Zholents, M. Zolotorev, A. Chin, R. Schoenlein, and C.V. Shank, Les Comptes Rendus de l'Académie des Sciences, Série IV, 1, 279-296 (2000).

[16] E. Esarey, P. Sprangle, J. Krall, and A. Ting, IEEE Trans. Plasma Sci. PS-24, 252 (1996).

[17] E. Esarey, J. Krall and P. Sprangle, Phys. Rev. Lett., 72, 2887 (1994).

[18] E. Esarey et al., J. Quant. Electron., 33, 1879-1914 (1997).

[19] A.Modena, Z.Najmudin, A.E.Dangor, C.E.Clayton, K.A.Marsh, C.Joshi, V. Malka, C.B.Darrow, C.Danson, D.Neely, and F.N.Walsh, Nature 377 ,606 (1995).

[20] K.Nakajima, D.Fisher, T.Kawakubo, H.Nakanishi, A.Ogata, Y.Kato, Y.Kitagawa, R.Kodama, K.Mima, H.Shiraga, K.Suzuki, K.Yamakawa, T.Zhang, Y.Sakawa, T. Shoji, Y.Nishida, N.Yugami, M.Downer, and T.Tajima, Phys. Rev. Lett. 74 , 4428 (1995).

[21] D. Umstadter, S.-Y. Chen, A. Maksimchuk, G. Mourou, and R. Wagner, Science 273, 472 (1996); R. Wagner, S.Y. Chen, A. Maksimchuk, and D. Umstadter, Phys.Rev.Lett. 78, 3125 (1997).

[22] A.Ting, C.I.Moore, K.Krushelnick, C.Manka, E.Esarey, P.Sprangle, R.Hubbard, 
H.R.Burris, and M.Baine, Phys. Plasmas 4, 1889 (1997); C.Moore, A.Ting, K.Krushelnick, E.Esarey, R.F.Hubbard, H.R.Burris, C.Manka, and P.Sprangle, Phys. Rev. Lett. 79, 3909 (1997).

[23] D.Gordon, K.C.Tzeng, C.E.Clayton, A.E.Dangor, V.Malka, K.A.Marsh, A. Modena, W.B.Mori, P.Muggli, Z.Najmudin, D.Neely, C.Danson, and C.Joshi, Phys. Rev. Lett. 80, 2133 (1998).

[24] F. Amiranoff, S. Baton, D. Bernard, B. Cros, D. Descamps, L.F. Dorchies, F. Jacquet, V. Malka, J.R. Marques, G. Matthieussent, P. Mine, A. Modena, P. Mora, J. Morillo, Z. Najmudin, Phys. Rev. Lett., 81, 995-8 (1998).

[25] F. Dorchies, F. Amiranoff, V. Malka, J.R. Marques, A. Modena, B. Cros, G. Matthussent, P. Mora, A. Solodov, J. Morillo, Z. Najmudin, Phys. Plasmas, 2903-13 (1999).

[26] M. I. K. Santala, Z. Najmudin, E. L. Clark, M. Tatarakis, K. Krushelnick, A. E. Dangor, V. Malka, J. Faure, R. Allott, and R. J. Clarke Phys. Rev. Lett. 86, 1227 (2001).

[27] W.P.Leemans, D.Rodgers, P.E.Catravas, C.G.R.Geddes, G.Fubiani, E.Esarey, B.A.Shadwick, R.Donahue, and A.Smith, Phys. Plasmas, to appear, June, 2001.

[28] E. Esarey, R. F. Hubbard, W. P. Leemans, A. Ting, and P. Sprangle, Phys. Rev. Lett. 79, 2682 (1997).

[29] C.B. Schroeder, P.B. Lee, J.S. Wurtele, E. Esarey, and W.P. Leemans, Phys. Rev. E 59, 6037 (1999).

[30] E. Esarey, C.B. Schroeder, W.P. Leemans, and B. Hafizi, Phys. Plasmas 6, 2262 (1999).

[31] D. Umstadter, J. K. Kim, and E. Dodd, Phys. Rev. Lett. 76, 2073 (1996).

[32] R. G. Hemker, K.-C. Tzeng, W. B. Mori, C. E. Clayton, and T. Katsouleas, Phys. Rev. E 57, 5920 (1998). 
[33] G. Fubiani, W. Leemans and E. Esarey, Submitted to Proc. of Advanced Accelerator Workshop, Santa Fe, NM, June, 2000.

[34] G.A. Mourou, C.P.J. Barty,M.D. Perry, Physics Today, 51, p. 22-28, January 1998. 